\title{
Evacuation Path Selection for Firefighters Based on Dynamic Triangular Network Model
}

\author{
Qianyi Zhang \\ College of Information Science and \\ Technology, Donghua University \\ Shanghai, china
}

\author{
Demin Li \\ College of Information Science and \\ Technology, Donghua University \\ Shanghai, china
}

\author{
Yajuan An \\ College of Information Science and \\ Technology, Donghua University \\ Shanghai, china
}

\begin{abstract}
Path selection is one of the critical aspects in emergency evacuation. In a fire scene, how to choose an optimal evacuation path for firefighters is a challenging aspect. In this paper, firstly, a dynamic triangular network model formed by robots is presented. On the basis of this model, directed graph is established in order to calculate direct paths. Then multiparameter information fusion which includes smoke density, temperature and oxygen density is discussed in detail for environment safety evaluation. Based on the discussions, a new way has been proposed for optimal path selection, taking into consideration the safety-factor of the path. The objectives of the method are to minimize the path lengths, at the same time, to protect firefighters from the dangerous regions. In the end, numerical simulation results prove the feasibility and superiority of this method.
\end{abstract}

Keywords- Evacuation Path Selection; Information Fusion; Dynamic Triangular Network Model.

\section{INTRODUCTION}

In recent years, frequent fire disasters have brought great casualties and huge property loss. Firefighter becomes a highrisk profession. When firefighters finish the search and rescue missions, the situation of the fire scene usually gets worse, meanwhile, the oxygen reserves the firefighters carried may become exhausted. Therefore the firefighters must evacuate to safety areas as soon as possible. Due to the complex, variable and uncertain situation, it is difficult for firefighters to orientate themselves and find a suitable path to the exit. How to choose an optimal evacuation path for firefighters is an important and challenging problem.

There are various researches regarding the problem of path selection in emergency situations. Marina Yusoff provides a good review on the mathematical algorithm and model of evacuation problems [1]. The ant colony optimization algorithm is applied to find the shortest path for emergence evacuation [2-4]. Tianyu Wang established a fire evacuation system and combined it with the Dijkstra Algorithm [5]. A multi-objective optimization model based genetic algorithm is adopted to solve the proposed evacuation routing problem [6]. A multi-objective approach is presented to identify evacuation paths and the location of shelters for urban evacuation planning [7].

By now, most researches only take the shortest path as the objective. However, the optimal path does not necessarily mean the shortest path. It should also take the path safety into consideration. Ulf Witkowski[8] put forward a dynamic triangular ad-hoc network formed by multi-robot systems to provide robust communication links for human and robots in a fire scene. Base on the dynamic triangular network model, Demin Li, etc. [9] proposed a method for establishing smoke density gradient based directed graph and selecting the shortest directed path for firefighters, but did not take the multiple parameter information fusion into account.

In this paper, we establish a new source-destination based directed graph in the dynamic triangular network model. Besides, Smoke density, temperature and oxygen density are considered in the information fusion for environment safety evaluation. Our objective is to minimize the path length, meanwhile, to make assure the firefighters keep away from the dangerous regions.

The paper is organized as follows: In section II, a dynamic triangular network model formed by multi-robots is established in a fire scene. In section III, the sourcedestination directed graph in the dynamic triangular network model is presented and described in detail. In section IV, firstly, multiple parameters consisting of temperature, smoke density and oxygen density are considered in information fusion process. The results of fusion are used to classify the environment into three safety levels. Secondly, based on the discussion all above, a new path selection method with safetyfactor considered is proposed and verified. The last section makes the conclusion.

\section{DYNAMIC TRIANGULAR NETWORK MODEL}

The modern buildings tend to be large-scale and complex at present. When a fire happens, all communication patterns that rely on infrastructures might be useless and cannot supply enough surrounding information for firefighters. Firefighters usually equip with multiple sensors and could get real-time information.

However, some regions are too dangerous for human to approach, the information collection is difficult. Paper [8] proposes a dynamic triangular method for robots distribution in a fire scene. All the robots are equipped with sensors to collect surrounding information and position data. The main purpose of the method is to deploy robots in a way with the largest coverage for facilitate communication and environment exploration. 


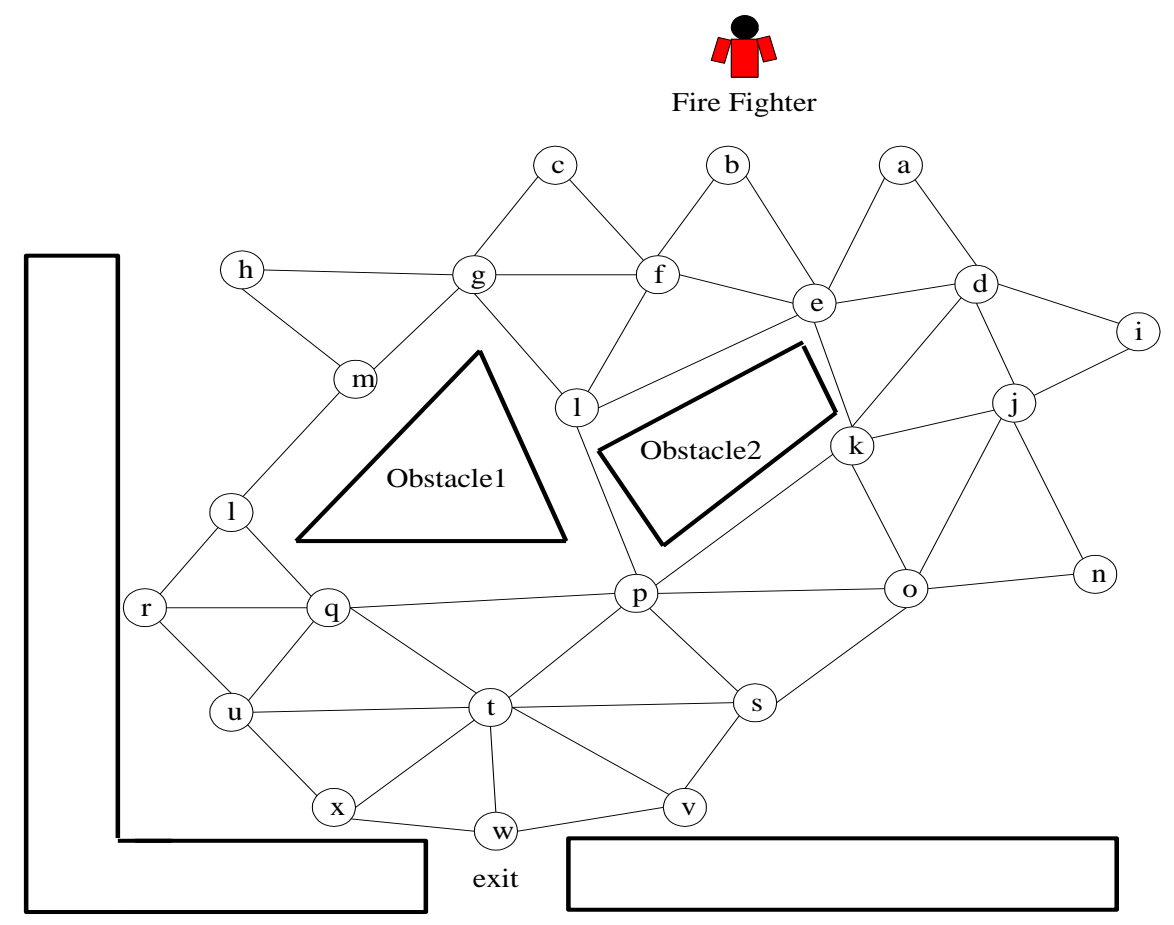

Figure.1. Dynamic triangular network model graph [9]

First of all, an ad-hoc network of robots needs to be built up. Ulf Witkowski provides a dynamic triangular network schema [8], based on this scheme, Demin Li, etc. proposed the dynamic triangular network model [9], it is shown in Figure 1.

The Figure 1 shows an environment covered by the ad-hoc network of robots using dynamic triangular network model. Robots act as the nodes of the network. Robots are represented as circles, marked by English letters. The communication links of the robots are indicated by solid line segments. The thicker solid lines in the figure represent the obstacles. The dynamic triangulation method provides positioning of beacons used as reference points at the vertices of equilateral or nearly equilateral triangles.

Some robots can be placed as beacons at the important position such as entrances, doors, corners and so on. Other robots might be distributed as uniformly as possible in order to gather reliable information about the environment. The robots will form a partition of the environment, separating it in regions, which will represent a triangulation in the absence of obstacles.

As shown in Figure 1, a fire fighter is going to evacuate to the exit. There are obstacles in the building, so it is not safe for him to run directly to the exit. Besides, the smoke and flames are also great threat to him. Since obstacles are surrounded by robots, and every robot could get the information of neighboring environment, it is wise to evacuate along the connection line between the robots. In this way, firefighters could avoid obstacles and high-risk region.

\section{ESTABLISHING DiRECTED GRAPH BASED ON DYNAMIC TRIANGULAR NETWORK MODEL}

To make the optimal path selection, firstly, directed graph in the dynamic triangular network model must be set up, so that all the feasible direct paths could be found out. The sub graph Figure 2 of Figure 1 shows 7 nodes in the network including the exit.

Node $\mathrm{Ri}$ is defined as a two dimension coordinate $(\mathrm{Xi}, \mathrm{Yi})$. $\mathrm{Xi}$ and $\mathrm{Yi}$ are horizontal coordinate and vertical coordinate of the node, respectively. Suppose $\mathrm{Rj}$ is the neighbor node of $\mathrm{Ri}$, Rs is the source node and Ro is the destination node (exit). We define that:

(1) If $|\arctan [(\mathrm{Yj}-\mathrm{Yi}) /(\mathrm{Xj}-\mathrm{Xi})]-\arctan [(\mathrm{Yo}-\mathrm{Ys}) /(\mathrm{Xo}-\mathrm{Xs})]|<90^{\circ}$, we could set the direction from Ri to $\mathrm{Rj}$;

(2) If $|\arctan [(\mathrm{Yj}-\mathrm{Yi}) /(\mathrm{Xj}-\mathrm{Xi})]-\arctan [(\mathrm{Yo}-\mathrm{Ys}) /(\mathrm{Xo}-\mathrm{Xs})]|>90^{\circ}$, we set the direction from $\mathrm{Rj}$ to $\mathrm{Ri}$;

(3) If $|\arctan [(\mathrm{Yj}-\mathrm{Yi}) /(\mathrm{Xj}-\mathrm{Xi})]-\arctan [(\mathrm{Yo}-\mathrm{Ys}) /(\mathrm{Xo}-\mathrm{Xs})]|=90^{\circ}$, the direction can be set from the further node(the distance between the node and the exit ) to the nearer node.

In this way we make sure that every movement of the firefighter is getting closer to the exit. According to the principle, we establish all the possible paths from node $\mathrm{b}$ to node $\mathrm{p}$. As shown in the figure, Vector Vef is perpendicular to Vbw, but Re is closer to Rw, so the direction should be set from $\mathrm{Rf}$ to $\mathrm{Re}$. 


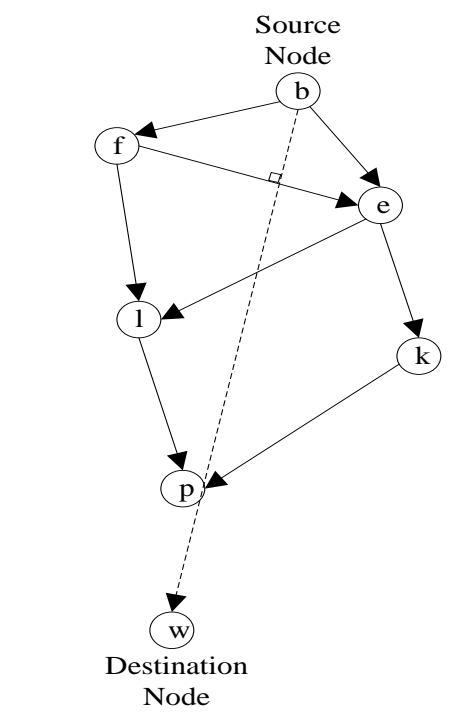

Figure 2. Directed graph from node $b$ to $w$

The directed graph can be converted into a path matrix $\mathrm{Cv}$ . If the directed arc is between the ith vertex and the jth vertex, the element of the ith row and jth column $\mathrm{C}(\mathrm{i}, \mathrm{j}) \mathrm{v}$ is $\mathrm{Eij}=\exp (\mathrm{dij})$ (dij is the length of arc between $\mathrm{Ri}$ and $\mathrm{Rj}$ ); Otherwise it is 0 . The path matrix of Fig. 2 can be defined as:

$V=\left[\begin{array}{c}b \\ e \\ f \\ k \\ l \\ p\end{array}\right], \quad C_{V}=\left[\begin{array}{cccccc}0 & 0 & 0 & 0 & 0 & 0 \\ E_{b e} & 0 & E_{f e} & 0 & 0 & 0 \\ E_{b f} & 0 & 0 & 0 & 0 & 0 \\ 0 & E_{e k} & 0 & 0 & 0 & 0 \\ 0 & E_{e l} & E_{f l} & 0 & 0 & 0 \\ 0 & 0 & 0 & E_{k p} & E_{l p} & 0\end{array}\right]$

$C_{V}^{2}=\left[\begin{array}{cccccc}0 & 0 & 0 & 0 & 0 & 0 \\ E_{b f} E_{f e} & 0 & 0 & 0 & 0 & 0 \\ 0 & 0 & 0 & 0 & 0 & 0 \\ E_{b e} E_{e k} & 0 & E_{f e} E_{e k} & 0 & 0 & 0 \\ E_{b e} E_{e i}+E_{b f} E_{f i} & 0 & E_{f e} E_{e l} & 0 & 0 & 0 \\ 0 & E_{e k} E_{k p}+E_{e l} E_{l p} & E_{f l} E_{l p} & 0 & 0 & 0\end{array}\right]$

$C_{V}^{3}=\left[\begin{array}{cccccc}0 & 0 & 0 & 0 & 0 & 0 \\ 0 & 0 & 0 & 0 & 0 & 0 \\ 0 & 0 & 0 & 0 & 0 & 0 \\ E_{b f} E_{f e} E_{e k} & 0 & 0 & 0 & 0 & 0 \\ E_{b f} E_{f e} E_{e l} & 0 & 0 & 0 & 0 & 0 \\ E_{b e} E_{e k} E_{k p}+E_{b e} E_{e l} E_{l p}+E_{b f} E_{f l} E_{l p} & 0 & E_{f e} E_{e k} E_{k p}+E_{f e} E_{e l} E_{l p} & 0 & 0 & 0\end{array}\right]$

$C_{V}^{4}=\left[\begin{array}{cccccc}0 & 0 & 0 & 0 & 0 & 0 \\ 0 & 0 & 0 & 0 & 0 & 0 \\ 0 & 0 & 0 & 0 & 0 & 0 \\ 0 & 0 & 0 & 0 & 0 & 0 \\ 0 & 0 & 0 & 0 & 0 & 0 \\ E_{b f} E_{f e} E_{e k} E_{k p}+E_{b f} E_{f e} E_{e l} E_{l p} & 0 & 0 & 0 & 0 & 0\end{array}\right]$

$$
C_{V}^{5}=C_{V}^{6}=\left[\begin{array}{llllll}
0 & 0 & 0 & 0 & 0 & 0 \\
0 & 0 & 0 & 0 & 0 & 0 \\
0 & 0 & 0 & 0 & 0 & 0 \\
0 & 0 & 0 & 0 & 0 & 0 \\
0 & 0 & 0 & 0 & 0 & 0 \\
0 & 0 & 0 & 0 & 0 & 0
\end{array}\right]
$$

If $C^{r} V(6,1)=0$, we conclude that there is no path from $\mathrm{Rb}$ to Rp taking num=r hops; while if $C^{r}{ }_{V} \quad(6,1)>0$, there is at least one path exist between $\mathrm{Rb}$ and $\mathrm{Rp}$ with num=r hops. So all the possible paths of Fig. 2 can be obtained by the formula as follows:

$$
\begin{aligned}
C_{V}^{\sum_{V}(6,1)=\sum_{n=1}^{6} C_{V}^{n}(6,1)} & =E_{b e} E_{e k} E_{k p}+E_{b e} E_{e l} E_{l p}+E_{b f} E_{f l} E_{l p} \\
& +E_{b f} E_{f e} E_{e k} E_{k p}+E_{b f} E_{f e} E_{e l} E_{l p}
\end{aligned}
$$

The value of the first factor of equation(1), $E_{b e} E_{e k} E_{k p}=\exp \left(d_{b e}+d_{e k}+d_{k p}\right)$, fully indicates the length of the path $: \mathrm{Rb} \rightarrow \mathrm{Re} \rightarrow \mathrm{Rk} \rightarrow \mathrm{Rp}$.

So the smallest factor of the formula corresponds to the shortest path. However, the optimal path does not mean the shortest path. It should also take the safety of the path into consideration. 


\section{The Optimal Evacuation PATH SElEction}

In fire scenes, there are many factors that may threaten the safety of firefighters, such as high temperature, smoke, poisonous gas and so on. Therefore, environmental situation around the selected path is essential.

Firstly, in section 3, we proposed a method to establish source-destination based directed graph, so that all feasible paths can be found out; Secondly, information fusion is used to evaluate the safety of nodes in each path, the nodes safety level reflects surrounding environment situation; Finally, safety-factor considered optimal path selection method is presented.

\section{A. Evaluation of the environment safety based on D-S evidence theory}

The environment situation of the fire scene is a complex situation with the characteristics of dynamic, nonlinear and uncontrolled. At the same time, due to the inaccuracy of sensor and environmental noise, the information gathered by single sensor may be not reliable and complete. So the multisensor information fusion is very necessary, which is used to increase the data using rate and to advance the information reliability and error tolerance.

Currently, there are several methods of multi-sensor information fusion, such as Bayesian test, Kalman filter, expert system, neural networks, fuzzy sets, D-S evidence theory and so on.

However, there are many shortages which are difficult to overcome in the process of fusion by these methods, the large amount of data calculation limits the application of Bayesian test and Kalman filter, the shortcoming of fuzzy sets is being more sensitive to the object of noise in the fusion process. Compared to this algorithm, D-S evidence theory [10-14] is better than the traditional methods to grasp the unknown and uncertainty of the problems, meanwhile, it does not require the priori probability, resulting in having been widely used in multi-sensor information fusion.

There are many factors that may threaten the safety of firefighters in fire scenes; we choose three most representative parameters to evaluate the environment situation: smoke density, temperature and oxygen density. According to the results of the fusion, environment safety levels are divided into three levels (I, II, III), representing safe, and medium, risky respectively.

The table 1 below shows the each probability of environment situation, it obtained by the expert system [11].

TABLE.I. EACH PRobabiLITy Of ENVIRONMENT Situation [11]

\begin{tabular}{|c|c|c|c|c|}
\hline Parameter & Range & $\begin{array}{c}\text { Safe } \\
\text { (I) }\end{array}$ & $\begin{array}{c}\text { Medium } \\
\text { (II) }\end{array}$ & $\begin{array}{c}\text { Risky } \\
\text { (III) }\end{array}$ \\
\hline \multirow{3}{*}{ Temperature } & $>=800$ & 0.2 & 0.1 & 0.7 \\
\cline { 2 - 5 } & $200<\mathrm{T}<800$ & 0.3 & 0.4 & 0.3 \\
\cline { 2 - 5 } & $<=200$ & 0.7 & 0.1 & 0.2 \\
\hline
\end{tabular}

\begin{tabular}{|c|c|c|c|c|}
\hline \multirow{3}{*}{$\begin{array}{l}\text { Smoke } \\
\text { Density }\end{array}$} & $>=1000$ & 0.2 & 0.1 & 0.7 \\
\cline { 2 - 5 } & $600<\mathrm{S}<1000$ & 0.3 & 0.3 & 0.4 \\
\cline { 2 - 5 } & $<=600$ & 0.7 & 0.1 & 0.2 \\
\hline \multirow{2}{*}{$\begin{array}{l}\text { Oxygen } \\
\text { Density }\end{array}$} & $<=14 \%$ & 0.2 & 0.1 & 0.7 \\
\cline { 2 - 5 } & $14 \%<02<21 \%$ & 0.3 & 0.4 & 0.3 \\
\cline { 2 - 5 } & $>=21 \%$ & 0.7 & 0.1 & 0.2 \\
\hline
\end{tabular}

If the information sensor gathered at the certain time is: $\mathrm{T}=400^{\circ} \mathrm{c}, \mathrm{S}=1200 \mathrm{ppm}, \mathrm{O}_{2}=10 \%$, Mi means different kinds of sensors, M(i) means different levels of environment situation. The probability distribution is as follow:

TABLE.II. Probability Distribution AT A CERTAin Time

\begin{tabular}{|c|c|c|c|}
\hline Probability & M(I) & M(II) & M(III) \\
\hline M1 & 0.3 & 0.4 & 0.3 \\
\hline M2 & 0.2 & 0.1 & 0.7 \\
\hline M3 & 0.2 & 0.1 & 0.7 \\
\hline
\end{tabular}

We obtain the results of information fusion with the algorithm of D-S evidence theory. $H(1)=M(1) \oplus M(2)$, $H(2)=M(1) \oplus M(2) \oplus M(3)$. Papers [13-14] provide a detailed definition and fusion steps of D-S evidence theory. Learning from that, we can obtain the fusion results shown in table III.

TABLE.III. RESULTS OF INFORMATION FUSION

\begin{tabular}{|c|c|c|c|}
\hline Probability & $\mathrm{M}(\mathrm{I})$ & $\mathrm{M}(\mathrm{II})$ & $\mathrm{M}(\mathrm{III})$ \\
\hline $\mathrm{H} 1$ & 0.2564 & 0.0256 & 0.718 \\
\hline M3 & 0.2 & 0.1 & 0.7 \\
\hline H2 & 0.0662 & 0.0009 & 0.9329 \\
\hline
\end{tabular}

According to all the tables above, the probabilities of level I, II, III are 0.0662 、 0.0009 、 0.9329 , $\max \{\mathrm{M}(\mathrm{I}), \mathrm{M}(\mathrm{II}), \mathrm{M}(\mathrm{III})\}$ is $\mathrm{M}$ ( III ) , obviously, the surrounding situation of the path is very dangerous, it warns the firefighters to avoid the high-risk node in the evacuation process. We can obtain environment safety level of each node by information fusion in order to provide for optimal path selection.

\section{B. Safety-factor considered optimal path selection}

Based on the results in section A, environment safety level of each node can be classified into a certain safety level through information fusion.

We assume that green, yellow and red circles represent safe, medium and risky nodes respectively. The directed graph with safety-factor weight is shown in Figure 3 as follows. 


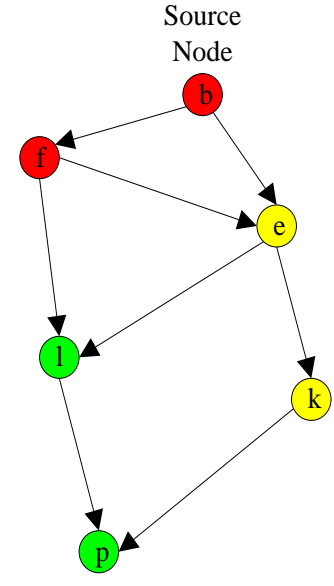

Figure 3. Directed graph with safety-factor weight

The node $\mathrm{Ri}$ can be set as a three dimension coordinate

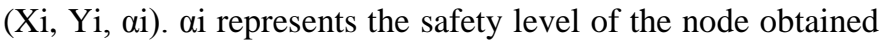
by information fusion above. $\alpha$ i adjusts the distance between nodes according to the safety level of the destination node. The coefficient $\alpha \mathrm{i}$ is larger when the safety level of destination node is higher. The value of $\alpha \mathrm{i}$ is assigned as a, 2a, 3a for level I (safe), II (medium) and III (risky) respectively. So we define:

$$
\mathrm{Dij}=\alpha \mathrm{i} * \mathrm{dij}
$$

Eij=exp(Dij), written as $E_{i j}^{\alpha_{i}}$

The path matrix evolves as follows:

$$
C_{V}=\left[\begin{array}{cccccc}
0 & 0 & 0 & 0 & 0 & 0 \\
E_{b e}^{\alpha_{e}} & 0 & E_{f e}^{\alpha_{e}} & 0 & 0 & 0 \\
E_{b f}^{\alpha_{f}} & 0 & 0 & 0 & 0 & 0 \\
0 & E_{e k}^{\alpha_{k}} & 0 & 0 & 0 & 0 \\
0 & E_{e l}^{\alpha_{l}} & E_{f l}^{\alpha_{l}} & 0 & 0 & 0 \\
0 & 0 & 0 & E_{k p}^{\alpha_{p}} & E_{l p}^{\alpha_{p}} & 0
\end{array}\right]
$$

According to formula (1), we can deduce that the safetyfactor path collections are as follows:

$$
\begin{aligned}
C_{V}^{\sum_{V}}(6,1)=\sum_{n=1}^{6} C_{V}^{n}(6,1) & =E^{\alpha_{e}}{ }_{b e} E^{\alpha_{k}}{ }_{e k} E^{\alpha_{p}}{ }_{k p}+E^{\alpha_{e}}{ }_{b e} E^{\alpha_{l}}{ }_{e l} E^{\alpha_{p}}{ }_{l p}+E^{\alpha_{f}}{ }_{b f} E^{\alpha_{l}}{ }_{f l} E^{\alpha_{p}}{ }_{l p} \\
& +E^{\alpha_{f}}{ }_{b f} E^{\alpha_{e}}{ }_{f e} E^{\alpha_{k}}{ }_{e k} E^{\alpha_{p}}{ }_{k p}+E^{\alpha_{f}}{ }_{b f} E^{\alpha_{e}}{ }_{f e} E^{\alpha_{l}}{ }_{e l} E^{\alpha_{p}}{ }_{l p}
\end{aligned}
$$

\section{Numerical simulation}

In order to verify the method presented above, we define the parameter of Figure 3 in Table IV \& Table V. The safety level of each node have obtained by information fusion has shown in table IV. Each path length we assumed and the comparison are listed in Table V.

\begin{tabular}{|c|c|c|c|}
\hline Arc & Length/m & Adjust Arc & length/m \\
\hline dbe & 5 & Dbe $=\alpha e$ dbe & $2 a * 5$ \\
\hline $\mathrm{dbf}$ & 5 & $\mathrm{Dbf}=\alpha \mathrm{f} \mathrm{dbf}$ & $3 a * 5$ \\
\hline dek & 4 & Dek $=\alpha \mathrm{k}$ dek & $2 \mathrm{a} * 4$ \\
\hline del & 8 & Del $=\alpha \mathrm{l}$ del & $a * 8$ \\
\hline $\mathrm{dfe}$ & 7 & $\mathrm{Dfe}=\alpha \mathrm{e}$ dfe & $2 a * 7$ \\
\hline $\mathrm{dfl}$ & 5 & $\mathrm{Dfl}=\alpha \mathrm{l} \mathrm{dfl}$ & $a * 5$ \\
\hline $\mathrm{dkp}$ & 8 & $\mathrm{Dkp}=\alpha \mathrm{p} \mathrm{dkp}$ & $a * 8$ \\
\hline $\mathrm{dlp}$ & 5 & $\mathrm{Dlp}=\alpha \mathrm{p} \mathrm{dlp}$ & $a * 5$ \\
\hline
\end{tabular}

TABLE.IV. SAFETY LEVEL OF EACH NODE

\begin{tabular}{|c|c|c|}
\hline Node & Safety Level & $\alpha \mathrm{i}$ \\
\hline $\mathrm{b}$ & III & $3 \mathrm{a}$ \\
\hline $\mathrm{e}$ & $\mathrm{II}$ & $2 \mathrm{a}$ \\
\hline $\mathrm{f}$ & $\mathrm{III}$ & $3 \mathrm{a}$ \\
\hline $\mathrm{k}$ & $\mathrm{II}$ & $2 \mathrm{a}$ \\
\hline $\mathrm{l}$ & $\mathrm{I}$ & $\mathrm{a}$ \\
\hline $\mathrm{p}$ & $\mathrm{I}$ & $\mathrm{a}$ \\
\hline
\end{tabular}

TABLE.V. The Distance BetweEn NoDES

From Table V, we can learn that the distance between nodes have changed after adjustment. The lengths of all feasible paths can be obtained by adding distances between nodes. The results are shown in Table VI as follows:

TABLE.VI. THE COMPARISON OF PATH LENGTHS BEFORE AND AFTER ADJUSTMENT

\begin{tabular}{|c|c|c|}
\hline Path & $\begin{array}{c}\text { Before } \\
\text { Adjust/m }\end{array}$ & After adjust/m \\
\hline $\mathrm{b} \rightarrow \mathrm{e} \rightarrow \mathrm{k} \rightarrow \mathrm{p}$ & 17 & $26 \mathrm{a}$ \\
\hline $\mathrm{b} \rightarrow \mathrm{e} \rightarrow \mathrm{l} \rightarrow \mathrm{p}$ & 18 & $23 \mathrm{a}$ \\
\hline $\mathrm{b} \rightarrow \mathrm{f} \rightarrow \mathrm{l} \rightarrow \mathrm{p}$ & 15 & $25 \mathrm{a}$ \\
\hline $\mathrm{b} \rightarrow \mathrm{f} \rightarrow \mathrm{e} \rightarrow \mathrm{k} \rightarrow \mathrm{p}$ & 24 & $45 \mathrm{a}$ \\
\hline $\mathrm{b} \rightarrow \mathrm{f} \rightarrow \mathrm{e} \rightarrow \mathrm{l} \rightarrow \mathrm{p}$ & 25 & $42 \mathrm{a}$ \\
\hline
\end{tabular}

According to Table VI, without safety consideration, the shortest path is $\mathrm{b} \rightarrow \mathrm{f} \rightarrow \mathrm{l} \rightarrow \mathrm{p}$, considering safety factor, the shortest path changes to $\mathrm{b} \rightarrow \mathrm{e} \rightarrow \mathrm{l} \rightarrow \mathrm{p}$. Noted that $\mathrm{f}$ is a high risk node, it is reasonable to avoid it. Therefore the Safety-factor considered optimal path selection method is superior to the old one. 


\section{CONCLUSIONS}

We have proposed a method of optimal evacuation path selection for firefighters based on the dynamic triangular network model. The network consists of robots who can work in unknown environment where information gather is difficult. Robots in the network are equipped with sensors to provide real-time information for firefighters. In order to find out all the feasible direct paths, we set up the directed graph by calculating the included angle between the vector of sourcedestination and the vector of two random nodes. In this way we make sure every movement is closer to the exit. For the multi-parameter information fusion, D-S evidence theory is applied to make accurate estimation of the environment, avoiding the misjudgment of single sensor. The objectives of the method are to minimize the path length for shortening evacuation time, meanwhile, to keep the firefighters from the dangerous regions.

In this paper, we assumed that there is only one exit in the dynamic triangular network model. Evacuation problem of multi-exit should also be explored. Besides, the positions of robots who gather information in the model are fixed. Actually, the robots may move with the firefighters in order to provide real-time information. A new model and an improved algorithm for evacuation path selection will be the future research directions of the authors.

\section{ACKNOWLEDGMENT}

This work is supported by the National Natural Science Foundation of China (NSFC) granted number 71171045.

\section{REFERENCES}

[1] Marina Yusoff, Junaidah Ariffin, Azlinah Mohamed, "Optimization Approaches for Macroscopic Emergency Evacuation Planning," A Survey, Information Technology, pp. 1-7, March 2008.

[2] Yuan Yuan, Dingwei Wang, "Multi-Objective Path Selection Model and Algorithm Emergency Evacuation," Proceedings of the IEEE International Conference on Automation and Logistics, 2007.
[3] Xinlu Zong, Shengwu Xiong., "Multi-objective Ant Colony Optimization Model for Emergency Evacuation," International Conference on Natural Computation, 2010.

[4] Zhixiang Fang, Xinlu Zong, Qingquan Li, QiupingLi, Shengwu Xiong. "Hierarchical multi-objective evacuation routing in stadium using ant colony optimization approach," Journal of Transport Geography. vol.19, pp. 443-451, 2007.

[5] Wang Tian-yu , Huang Rui, "The Application of the Shortest Path Algorithm in the Evacuation System," International Conference of Information Technology, Computer Engineering and Management Sciences, 2011

[6] Qiuping Li, Zhixiang Fang, Qingquan Li, "Multi-objective Evacuation Route Assignment Model Based on Genetic Algorithm," Geoinformatics, 18th International Conference, 2010.

[7] João Coutinho-Rodrigues, Lino Tralhão, Luís Alçada-Almeida, "Solving a location-routing problem with a multi-objective approach: the design of urban evacuation plans," Journal of Transport Geography. vol.22, pp. 206-218, 2012.

[8] Ulf Witkowski, Mohamed EI-Habbal, "Ad-hoc net- work communication infrastructure for multi-robot systems in disaster scenarios," Proceedings of the EURON/IARP International Workshop on Robotics for Risky Interventions and Surveillance of the Environment, 2008.

[9] Demin Li, Jie Zhou, Jiacun Wang, Yun Wang, Qianyi Zhang, “ Information Fusion and Path Selection for Firefighters Based on Ad Hoc Dynamic Triangular Network," International Journal of Advancements in Computing Technology, unpulished..

[10] Rombaut M, Zhu YM, "Study of Dempster-Shafer for image segmentation applications," Image Vis Computer. vol. 20, pp.15-23, 2002

[11] D.L.Hall, "Mathematical Techniques in Multi-sensor Data Fusion," Boston, London: Artech House, 1992.

[12] Li Xiangli, Lv Jianping, "Improvement of D-S Theory of Evidence in Multi-sensor Information Fusion System," Sensor Technology, 2005.

[13] Li Yi-Bo, Wang Ning, "Based on D-S Evidence Theory of Information Fusion Improved Method," International Conference on Computer Application and System Modeling, 2010.

[14] Jia-nan Chu, Yunhua Li, "Study on the Coal Mine Safety Assessment Based on Rough Set-Neural Network-Evidence Theory." International Conference on Future Information Technology and Management Engineering, 2010 